\title{
Equilibrium and stability properties of detonation waves in the hydrodynamic limit of a kinetic model
}

\author{
Wilson Marques Jr. ${ }^{a}{ }^{*}$, Ana Jacinta Soares ${ }^{b)}$, Miriam Pandolfi Bianchi $\left.^{c}\right) \ddagger$ \\ Gilberto M. Kremer ${ }^{a)}$, \\ ${ }^{a)}$ Departamento de Física, Universidade Federal do Paraná, Curitiba, Brazil \\ ${ }^{b)}$ Centro de Matemática, Universidade do Minho, Braga, Portugal \\ c) Dipartimento di Matematica, Politecnico di Torino, Torino, Italy
}

\begin{abstract}
A shock wave structure problem, as the one which can be formulated for the planar detonation wave, is analyzed here for a binary mixture of ideal gases undergoing the symmetric reaction $\mathrm{A}_{1}+\mathrm{A}_{1} \rightleftharpoons \mathrm{A}_{2}+\mathrm{A}_{2}$. The problem is studied at the hydrodynamic Euler limit of a kinetic model of the reactive Boltzmann equation. The chemical rate law is deduced in this frame with a second-order reaction rate, in a chemical regime such that the gas flow is not far away from the chemical equilibrium. The caloric and the thermal equations of state for the specific internal energy and temperature are employed to close the system of the balance laws. With respect to other approaches known in the kinetic literature for detonation problems with a reversible reaction, this paper is addressed to improve some aspects of the wave solution. Within the mathematical analysis of the detonation model, the equation of the equilibrium Hugoniot curve of the final states is explicitly derived for the first time and used to define the correct location of the equilibrium Chapman-Jouguet point in the Hugoniot diagram. The parametric space is widened to investigate the response of the detonation solution to the activation energy of the chemical reaction. At last, the mathematical formulation of the linear stability problem is given for the wave detonation structure via a normal-mode approach, when bidimensional disturbances perturb the steady solution. The stability equations with their boundary conditions and the radiation condition of the considered modeling are explicitly derived for small transversal deviations of the shock wave location. The paper shows how a second-order chemical kinetics description, derived at the microscopic level, and an analytic deduction of the equilibrium Hugoniot curve, lead to an accurate picture of the steady detonation with reversible reaction, as well as to a proper bidimensional linear stability analysis.
\end{abstract}

PACS: 47.70.Fw 47.40.Rs 82.40.Fp 47.20.-k

Keywords: Chemically reactive flows. Detonation waves. Hyperbolic systems. Hydrodynamic stability

\footnotetext{
*marques@fisica.ufpr.br

†ajsoares@math.uminho.pt

${ }^{\ddagger}$ miriam.pandolfi@polito.it

§kremer@fisica.ufpr.br
} 


\section{Introduction}

Among several shock wave structure problems arising in hyperbolic systems described by balance laws, the one which deals with the detonation in reactive gaseous mixtures is perhaps the most frequently investigated. The interest is addressed both to the evolution dynamics of the detonation wave, and to the attempts of a comprehensive understanding of the rather complex physical phenomenology of combustion processes, see for instance $[1,2]$ and related bibliography.

The renewed interest in detonation phenomena, even though investigated with simple models, is widely documented in literature, see for instance papers $[2,3,4]$. The motivation of this interest is essentially due to the development of detonation wave engines and explosives modelling, to the safety issues and estimation of explosion, and also to the numerical modelling and combustion simulations. Recent contributions dealing with the wave structure in combustion processes, connected to the kinetic theory of reacting gases, can be found for an irreversible reaction in papers $[5,6,7]$, and for a reversible reaction in papers $[8,9,10,11]$. In particular, the hydrodynamic stability of the detonation wave is examined in papers $[10,11]$. Microscopic simulations of wave fronts and transition from different regimes to detonation are developed for example in papers $[12,13]$. Other involved works of reactive flows in the framework of the kinetic theory should also be quoted for what concerns the non equilibrium kinetics behind a strong shock wave, as for example papers [14, 15].

A combustion-driven shock wave that propagates in a supersonic regime with respect to an unburnt gas mixture, and which is sustained by the energy release of an exothermic chemical reaction, typically represents a detonation wave. The hyperbolic reactive Euler equations, given by the conservation laws for mass, momentum and energy of the mixture, and by the chemical rate equation, constitute the governing equations and provide a simple and mathematically tractable model, which is commonly used as the starting step towards the characterization of the shock wave structure and its hydrodynamic stability. The governing equations of the detonation process admit steady solutions. In the shock attached coordinate system, the Rankine-Hugoniot relations derived from the conservation laws, together with the chemical rate equation, describe the distribution of pressure, velocity and mass density in the reaction zone.

The significant elements which allow one to distinguish and classify the many approaches to the detonation wave problem cover: type of chemical reaction, model of the chemical rate, account of the framework where the analysis is developed, level of the closure procedure, choice of the constitutive equations, and at last consideration of the specific thermodynamical assumptions.

The present study is addressed to investigate both the shock structure problem and bidimensional linear stability for detonation waves arising in a binary reacting gas mixture, when the constituents undergo a symmetric reversible explosive chemical reaction of type $A_{1}+A_{1} \rightleftharpoons A_{2}+A_{2}$. The chemical rate law, specifying the evolution of the progress of the considered reaction, is derived from the kinetic theory extended to reacting gases. The reaction rate results then to be of second order, namely it is proportional to the square of the product concentrations, see Ref. [16]. In particular, the rate law incorporates both the effects of the reactive binary encounters and the effects due to the specific reaction heat and forward activation energy. Moreover, the reactive mixture is considered to be Eulerian (i.e. a mixture where viscosity, heat conductivity and diffusive effects are negligible), and the specific heat 
ratios of the constituents are assumed to be constant. In this way, the thermal equation of state for the reactive mixture defines the pressure in dependence on the temperature and mass density of the mixture. The caloric equation of state defines the specific internal energy of the mixture as function of the specific heat ratio, specific reaction heat and progress variable of the chemical reaction, i.e. mass concentration of products. The rate law and the equations of state define the constitutive conditions for the reactive Euler equations.

The mathematical model proposed in this paper offers the capability of representing a realistic structure of the steady detonation wave and its instabilities, since it involves several basic parameters, as the detonation wave velocity, the specific reaction heat, the activation energy of the chemical reaction and, more in particular, the ratio of the specific heats. This rather wide spectrum allows to provide a rich description of the detonation dynamics, in the sense that a more complete picture can be obtained varying these parameters. In detail, the ratio of specific heats $\gamma$ accounts for the multi-component nature of the mixture, the reaction heat $\mathcal{Q}$ defines either the exothermic or the endothermic character of the reaction, the activation energy $\epsilon_{f}$ specifies the threshold of the forward chemical reaction, and the detonation velocity $s$ indicates the overdrive degree of the detonation wave.

The influence of the above mentioned parameters on the instability behaviour also covers an important role in view of an exhaustive stability analysis of the detonation wave solution. Thus a contribution is given with respect to previous approaches available in the kinetic literature of reacting gases, where fewer parameters are usually considered, see for example $[10,11]$. Moreover, a contribution can be extended also to the classical studies of detonation where, to the best of our knowledge, a second order reversible reaction does not seem to be considered.

At the same time, the use of a second order chemical rate, deduced at the microscopic level within the kinetic theory of reacting gases presented in the book [17], can be viewed as a refinement useful to exploit the chemical kinetics underlying the detonation process the stability analysis.

Furthermore, the mathematical modelling which characterizes this work permits to set up an accurate and complete Rankine-Hugoniot analysis, for what concerns the equilibrium properties of the detonation solution. The so called equilibrium Hugoniot curve, locus of all final states of the combustion process with reversible reaction, is here introduced for the first time in the context of the kinetic literature. Such curve is analytically derived in an explicit parametric form and then used to determine the equilibrium Chapman-Jouguet point. The procedure here proposed differs from the classical one, which is based on the Jouguet's rule and the Chapman-Jouguet condition formulated in terms of equilibrium sonic considerations $[1,18,19,20]$.

The paper is organized in six sections. Section 2 presents an useful preliminar content on the model reactive Euler equations and their properties. Attention is given to the reaction rate deduced from the kinetic theory in a second order form. In Section 3, a detailed RankineHugoniot analysis is developed within the steady detonation wave problem. The equilibrium properties of the detonation solution are investigated and the equilibrium Hugoniot curve is analytically derived. In Section 4, the structure of the detonation wave solution is analytically described on the basis of the Rankine-Hugoniot conditions and reactive Euler equations of the previous sections. The solution procedure is also presented. In Section 5, such procedure is applied in order to numerically solve the steady detonation problem. Two study cases 
are considered and some numerical studies are performed at the scope of investigating the influence of the chemical kinetic parameters on the detonation structure. At last, Section 6 deals with the bidimensional linear stability analysis of the steady solution. Using a normalmode approach, the boundary value problem for the stability equations is formulated and the radiation condition is constructed in detail. Its final form shows that it is possible to obtain, as a particular case, the radiation condition when a first-order reaction rate of Arrhenius type is adopted.

\section{The unidimensional model for the reactive gas mixture}

In the present study a chemically reacting binary mixture of ideal gases is considered, for which the constituents $A_{1}$ and $A_{2}$ undergo the symmetric reversible reaction $A_{1}+A_{1} \rightleftharpoons$ $A_{2}+A_{2}$. The constituents are characterized by the same molecular masses and diameters, namely $m_{1}=m_{2}=m, a_{1}=a_{2}=a$, as well as by the specific formation (or binding) energies $\epsilon_{1}$ and $\epsilon_{2}$.

\section{Balance equations}

In the thermodynamic theory of mixtures [21] each constituent is assumed to obey a set of balance laws. Such laws express the rates of change for mass, momentum and energy when the proper production terms arising from the mutual constituent interaction are considered. Here the evolution equations of interest are those for the mixture mass density $\rho$, the mixture flow velocity $u$, the mixture pressure $p$ and the progress variable $z=\rho_{2} / \rho$ - defined as the mass concentration of the products - of an Eulerian reacting mixture. In the one-dimensional case they can be written as

$$
\begin{aligned}
& \frac{\partial \rho}{\partial t}+\frac{\partial}{\partial x}(\rho u)=0, \\
& \frac{\partial \rho u}{\partial t}+\frac{\partial}{\partial x}\left(\rho u^{2}+p\right)=0, \\
& \frac{\partial \rho e}{\partial t}+\frac{\partial}{\partial x}(\rho e u+p u)=0, \\
& \frac{\partial z}{\partial t}+u \frac{\partial z}{\partial x}=r
\end{aligned}
$$

where $e=\varepsilon+u^{2} / 2$ is the total specific energy of the mixture and $\varepsilon=\varepsilon(\rho, p, z)$ is the specific internal energy of the mixture. Moreover, $r=r(\rho, p, z)$ denotes the reaction rate whose explicit form is given below.

Equations of state

By assuming a single temperature $T$ for the reactive mixture with equal specific heat ratios $\gamma$ for both constituents, the pressure $p_{\alpha}$, the mass density $\rho_{\alpha}$ and the specific internal energy $\varepsilon_{\alpha}$ of each constituent obey the following thermal and caloric equations of state

$$
p_{\alpha}=\rho_{\alpha} \frac{k}{m} T \quad \text { and } \quad \varepsilon_{\alpha}=\epsilon_{\alpha}+\int_{T_{0}}^{T} c_{v}^{\alpha}(T) d T
$$

where $k$ denotes the Boltzmann constant, $c_{v}^{\alpha}(T)$ is the specific heat at constant volume for each constituent and the index zero refers to a reference state. Under the assumption that 
all chemical species have constant and equal specific heats $c_{v}$, the pressure and the specific internal energy of the mixture obey the following thermal and caloric equations of state

$$
p=\sum_{\alpha} p_{\alpha}=\rho \frac{k}{m} T \quad \text { and } \quad \varepsilon=\sum_{\alpha} \frac{\rho_{\alpha}}{\rho} \varepsilon_{\alpha}=\frac{p}{\rho(\gamma-1)}-z \Delta \epsilon,
$$

where $\epsilon_{1}=c_{v} T_{0}$ has been assumed for convenience sake, and $\Delta \epsilon=\epsilon_{1}-\epsilon_{2}$. Observe in particular that, due to the microscopic description of the chemical reaction, proper of the adopted chemical kinetics model, the ideal caloric equation of state shows a dependence on both the chemical progress variable and the specific reaction heat.

\section{Reaction rate}

The source contribution to the evolution of the progress variable $z$ is due to the chemical reaction and is specified resorting to the kinetic theory for chemically reactive mixtures. Accordingly, starting from a molecular collisional dynamics, the microscopic evolution of the mixture is described in terms of velocity distribution functions as well as elastic and reactive collisional operators. At the same time, the macroscopic properties of the gaseous mixture can be deduced and the corresponding governing equations can be derived in the considered hydrodynamic limit. The production terms are obtained integrating the collisional operators with prescribed elastic and reactive cross sections as well as pertinent distribution functions compatible with the assumed chemical regime (see, for example, book [17] for details).

For the reactive mixture considered in this paper the reaction rate is obtained in a flow regime close to the thermodynamical equilibrium, assuming reactive differential cross sections with activation energy and Maxwellian velocity distribution functions, which do not assure chemical equilibrium, of the form

$$
f_{\alpha}=n_{\alpha}\left(\frac{m}{2 \pi k T}\right)^{3 / 2} \exp \left(-\frac{m C^{2}}{2 k T}\right)
$$

where $n_{\alpha}=\rho_{\alpha} / m$ is the particle number density and $C$ the peculiar velocity of each constituent. Such Maxwellians do not assure, in general, the chemical equilibrium. Adopting reactive differential cross sections for binary collisions of hard spheres with activation energy, and taking into account the indistinguishability of the colliding particles, the reaction rate results in the form

$$
r=2 n \sqrt{\frac{\pi k T}{m}} a^{2} \exp \left(-\frac{\epsilon_{f}}{k T}\right)\left[(1-z)^{2}-z^{2} \exp \left(\frac{\epsilon_{f}-\epsilon_{r}}{k T}\right)\right],
$$

where $\epsilon_{f}$ and $\epsilon_{r}$ are the forward and backward activation energies. The reaction rate is the difference between a second-order forward rate proportional to the square of the $\mathrm{A}_{1}$ concentration, and a second-order backward rate proportional to the square of the $\mathrm{A}_{2}$ concentration. The chemical equilibrium condition implies

$$
\frac{1-z}{z}=\exp \left(\frac{\mathcal{Q}}{2 k T}\right)
$$

where $\mathcal{Q}=\epsilon_{f}-\epsilon_{r}=-2 m \Delta \epsilon$ is the reaction heat. 
Reactive Euler equations and their hyperbolicity

The governing equations (1-4), closed with the constitutive equations (6) and (8), constitute the reactive Euler equations of the mixture. They can be written in matrix form as

$$
\frac{\partial \mathbf{U}}{\partial t}+\frac{\partial \mathbf{F}(\mathbf{U})}{\partial x}=\mathbf{S}(\mathbf{U})
$$

where

$$
\mathbf{U}=\left[\begin{array}{c}
\rho \\
\rho u \\
\rho e \\
\rho z
\end{array}\right], \quad \mathbf{F}(\mathbf{U})=\left[\begin{array}{c}
\rho u \\
\rho u^{2}+p \\
\rho(e+p / \rho) u \\
\rho z u
\end{array}\right] \quad \text { and } \quad \mathbf{S}(\mathbf{U})=\left[\begin{array}{c}
0 \\
0 \\
0 \\
\rho r
\end{array}\right]
$$

In view of the hyperbolicity analysis of the reactive Euler equations, the matrix Eq. (10) is rewritten as

$$
\frac{\partial \mathbf{U}}{\partial t}+\mathbf{A}(\mathbf{U}) \frac{\partial \mathbf{U}}{\partial x}=\mathbf{S}(\mathbf{U})
$$

where

$$
\mathbf{A}(\mathbf{U})=\frac{\partial \mathbf{F}(\mathbf{U})}{\partial \mathbf{U}}=\left[\begin{array}{cccc}
0 & 1 & 0 & 0 \\
-\frac{(3-\gamma)}{2} u^{2} & (3-\gamma) u & (\gamma-1) & (\gamma-1) \Delta \epsilon \\
\frac{(\gamma-1)}{2} u^{3}-H u & H-(\gamma-1) u^{2} & \gamma u & (\gamma-1) \Delta \epsilon u \\
-z u & z & 0 & u
\end{array}\right] .
$$

Here, $H$ is the total specific enthalpy defined as

$$
H=e+\frac{p}{\rho}=\gamma e-\frac{(\gamma-1)}{2} u^{2}+(\gamma-1) z \Delta \epsilon .
$$

The characteristic velocities of the system (1-4) are the eigenvalues $\lambda_{i}$ of the Jacobian matrix $\mathbf{A}(\mathbf{U})$ defined in Eq. (13). They are given by

$$
\lambda_{1}=u-c<\lambda_{2}=\lambda_{3}=u<\lambda_{4}=u+c,
$$

where

$$
c=\sqrt{(\gamma-1)\left(H-\frac{u^{2}}{2}+z \Delta \epsilon\right)}=\sqrt{\gamma \frac{p}{\rho}}
$$

is the adiabatic speed of sound. Moreover, it follows straightforward that the eigenvectors of the Jacobian matrix $\mathbf{A}(\mathbf{U})$ constitute a complete set and therefore the system of balance equations (1-4) is hyperbolic, as expected when the hydrodynamic equations are closed at the Euler level (see [22]). In this case, the single-temperature model for such Euler fluid can be regarded as a principal subsystem of a multi-temperature model in the sense of extended thermodynamics (see [23, 24]). 


\section{Steady formulation of the wave structure problem}

The hyperbolic system (1-4) of the reactive Euler equations admits propagating wave solutions connecting equilibrium states ahead and behind the shock. A particular case of interest is the steady detonation solution consisting of a plane shock wave (moving along the $x$-axis with constant velocity $s>0$ ), followed by the reaction zone with finite thickness.

\subsection{Rankine-Hugoniot analysis}

The steady solution is found by means of the coordinate transformation to the steady variable

$$
\xi=s t-x,
$$

which places the system of equations (1-4) in the detonation frame reference so that $\xi<0$ ahead of the shock and $\xi>0$ behind the shock. The mathematical procedure which leads to the steady detonation solution follows the classical approach $[1,18,19]$. By starting from the reactive Euler equations referred to the steady variable $\xi$ and the corresponding algebraic Rankine-Hugoniot $(\mathrm{RH})$ conditions, the solution procedure is detailed in this section with reference to the considered model. The qualitative description of the wave structure problem is also included with the aim of interpreting the key properties of the solution, and adapting them to the considered model.

\section{Rankine-Hugoniot conditions}

Wave propagation problems within a chemically reactive mixture are studied by means of the algebraic $\mathrm{RH}$ jump conditions together with the reaction rate equation (4). Such $\mathrm{RH}$ conditions are deduced from the conservation equations (1-3), after transforming to the steady variable (15). A formal integration of the resulting equations between the initial state of the undisturbed mixture

$$
\rho=\rho_{0}, \quad u=u_{0}=0, \quad p=p_{0}, \quad \varepsilon=\varepsilon_{0}, \quad z=z_{0}=0,
$$

just ahead the shock, and the final state, just behind the shock. leads to the RH conditions

$$
\begin{aligned}
& \rho w=\rho_{0} s, \\
& p+\rho w^{2}=p_{0}+\rho_{0} s^{2}, \\
& \varepsilon+\frac{p}{\rho}+\frac{w^{2}}{2}=\varepsilon_{0}+\frac{p_{0}}{\rho_{0}}+\frac{s^{2}}{2}
\end{aligned}
$$

where $w=s-u$ is the waveframe velocity. From the above RH conditions one can easily deduce the following equations which have a meaningful geometric interpretation in the context of the problem of the combustion wave structure.

Rayleigh lines

Considering mass and linear momentum equations (17) and (18), the family of the Rayleigh lines of the proposed model is obtained in the form

$$
p / p_{0}=1-s^{2}\left(v / v_{0}-1\right),
$$

where $v=1 / \rho$ is the specific volume of the mixture and $s$ is the dimensionless wave speed given in units of $\sqrt{p_{0} v_{0}}$. Usually, the dimensionless wave speed is given in the literature in 
units of the Mach number. Here, $s$ is related with the shock Mach number $M$ as $s=\sqrt{\gamma} M$. Each Rayleigh line contains the initial state and all states in the reaction zone between the post-shock state and the final state, reached for the corresponding value of $s$. Thus, the Rayleigh lines represent conservation of mass and momentum of the mixture in these states.

Hugoniot curves

By using relation (17) and taking into account the caloric equation $(6)_{2}$, one obtains from the energy conservation equation (19) the family of the Hugoniot curves of fixed product concentration $z$,

$$
\frac{p}{p_{0}}=\frac{2 z \Delta \epsilon+\frac{\gamma+1}{\gamma-1}-\frac{v}{v_{0}}}{\frac{\gamma+1}{\gamma-1} \frac{v}{v_{0}}-1}
$$

where $\Delta \epsilon$ is given in units of $p_{0} v_{0}$. Since the pressure must be positive, one requires that the following condition holds

$$
\frac{\gamma-1}{\gamma+1}<\frac{v}{v_{0}}<2 z \Delta \epsilon+\frac{\gamma+1}{\gamma-1}
$$

By introducing $\mu^{2}=(\gamma-1) /(\gamma+1)$, equation (21) can be written in the form

$$
\left(\frac{p}{p_{0}}+\mu^{2}\right)\left(\frac{v}{v_{0}}-\mu^{2}\right)=2 \mu^{2} z \Delta \epsilon+\left(1+\mu^{2}\right)\left(1-\mu^{2}\right)
$$

which clearly indicates that the Hugoniot curves are hyperbolas in the $(v, p)$-plane centered at the point $\left(p / p_{0}, v / v_{0}\right)=\left(-\mu^{2}, \mu^{2}\right)$. Note that equations (23) defines a family of Hugoniot curves parametrized by the product concentration $z \in[0,1]$. Each curve of this family is the locus of all states in the reaction zone with the same value of $z$ and represents conservation of total energy in all states belonging to the curve itself. In particular, the curve corresponding to $z=0$ is the unreacted Hugoniot curve of all states for which the chemical reaction has not yet started. The one corresponding to $z=1$ is the totally reacted Hugoniot curve of all states for which the reactants of the forward reaction have been transformed into products. This situation is typical of an irreversible chemical reaction, but it is not proper of a reversible chemical reaction. In the latter case, the reaction proceeds until the chemical equilibrium is reached for $z=z_{\mathrm{e}}<1$, where $z_{\mathrm{e}}$ varies with the mixture temperature according to the chemical equilibrium condition.

\subsection{Equilibrium properties}

When the reaction is reversible, the Hugoniot diagram, besides the Hugoniot hyperbolas for a fixed $z$ concentration, and besides the Rayleigh lines for a fixed $s$ velocity, contains the so called equilibrium Hugoniot curve, which is the locus of all equilibrium final states of a detonation wave. Since the final states have different concentrations $z_{e q}$ varying with the mixture temperature, the equilibrium Hugoniot curve does not belong to the family of the Hugoniot hyperbolas for fixed $z$ concentration. This aspect, well exploited in the classical literature [18, 19, 25, 20], was never recognized before in the kinetic studies of detonation with reversible reaction. In this subsection, the analytic representation of the equilibrium Hugoniot curve is obtained, the physically consistent equilibrium states are precisely determined and the solution domain is also detailed. 


\section{Equilibrium Hugoniot curve}

Considering the curves represented by equations (20) and (23), their intersection points represent the states in the $(v, p)$-plane where mass, linear momentum and total energy are conserved. Such states are characterized by means of the following expressions

$$
\begin{aligned}
& \frac{v}{v_{0}}=\frac{\gamma}{\gamma+1} \frac{1+s^{2}}{s^{2}} \mp \frac{\sqrt{\left(s^{2}-\gamma\right)^{2}-2(\gamma+1)(\gamma-1) z \Delta \epsilon s^{2}}}{(\gamma+1) s^{2}} \\
& \frac{p}{p_{0}}=\frac{1+s^{2}}{\gamma+1} \pm \frac{\sqrt{\left(s^{2}-\gamma\right)^{2}-2(\gamma+1)(\gamma-1) z \Delta \epsilon s^{2}}}{(\gamma+1)}
\end{aligned}
$$

The above expressions determine all states behind the shock wave in dependence on the product concentration $z$ for assigned wave speed $s$. The choice of the upper signs identifies a state compatible with a strong detonation wave, while the choice of the lower signs identifies a state compatible with a weak detonation wave (see [1] for details). In particular, expressions (24) and (25) define the final states in the reaction zone for a strong detonation, when $z$ is constrained to the equilibrium condition (9). Expressing $T$ in terms of $p$ and $v$ by means of the state equation $(6)_{1}$, such condition can be rewritten as

$$
\left(\frac{1+s^{2}}{\gamma+1}+s^{2} \beta(z)\right)\left(\frac{\gamma}{\gamma+1} \frac{1+s^{2}}{s^{2}}-\beta(z)\right)=-\frac{\Delta \epsilon}{\ln \left(\frac{1-z}{z}\right)}
$$

where

$$
\beta(z)=\frac{\sqrt{\left(s^{2}-\gamma\right)^{2}-2(\gamma+1)(\gamma-1) z \Delta \epsilon s^{2}}}{(\gamma+1) s^{2}} .
$$

Therefore, expressions (24) and (25), combined with the equilibrium condition (26), define the equilibrium Hugoniot curve, locus of all equilibrium final states of a strong detonation wave. Such curve represents conservation of mass, linear momentum and total energy of the mixture at the states of chemical equilibrium. In particular, under the conditions of the implicit function theorem, the equilibrium concentration can be defined from condition (26) as a function of the wave speed $s$, that is $z=z_{\mathrm{e}}(s)$. This means that the equations of the equilibrium Hugoniot curve can be obtained from (24), (25) and (26) in the form

$$
\frac{v}{v_{0}}=f\left(s, z_{\mathrm{e}}(s)\right) \quad \text { and } \quad \frac{p}{p_{0}}=g\left(s, z_{\mathrm{e}}(s)\right)
$$

where $f\left(s, z_{\mathrm{e}}(s)\right)$ and $g\left(s, z_{\mathrm{e}}(s)\right)$ denote the right-hand side of equations (24) and (25) referred to the equilibrium concentration $z_{\mathrm{e}}$,

$$
f\left(s, z_{\mathrm{e}}(s)\right)=\frac{\gamma}{\gamma+1} \frac{1+s^{2}}{s^{2}} \mp \beta\left(z_{\mathrm{e}}(s)\right) \quad \text { and } \quad g\left(s, z_{\mathrm{e}}(s)\right)=\frac{1+s^{2}}{\gamma+1} \pm s^{2} \beta\left(z_{\mathrm{e}}(s)\right) .
$$

Equations (28) and (29), with $s$ as parameter, constitute the parametric equations of the complete equilibrium Hugoniot curve. The choice of the upper signs in expressions (29) determines the strong detonation branch of the equilibrium Hugoniot curve, whereas the choice of the lower signs determines the weak detonation branch. 


\section{Equilibrium Chapman-Jouguet state}

The Chapman-Jouguet state (CJ) is the equilibrium final state obtained for the minimum acceptable value of the wave speed $s$ for which the corresponding Rayleigh line intersects the equilibrium Hugoniot curve. Such minimum value is the so-called Chapman-Jouguet velocity $s_{j}$ and it can be obtained as that value of $s$ for which the corresponding Rayleigh line is tangent to the equilibrium Hugoniot curve, so that their slopes must be equal at the tangency point. The slope of the equilibrium Hugoniot curve (EHC) can be obtained from the parametric equations (28) as

$$
\left.\frac{d p}{d v}\right|_{\mathrm{EHC}}=\frac{p_{0}}{v_{0}} \frac{\frac{d g}{d s}}{\frac{d f}{d s}}=\frac{p_{0}}{v_{0}} \frac{\frac{\partial g}{\partial s}+\frac{\partial g}{\partial z_{\mathrm{e}}} \frac{d z_{\mathrm{e}}}{d s}}{\frac{\partial f}{\partial s} \frac{d z_{\mathrm{e}}}{\partial z_{\mathrm{e}}}}
$$

where the partial derivatives of the functions $f$ and $g$ are computed by using expressions (29) with the upper signs, whereas the total derivative $d z_{\mathrm{e}} / d s$ is the derivative of the implicit function $z_{\mathrm{e}}(s)$ computed from equilibrium condition (26). In order to compute such derivative, the equilibrium condition (26) is first rewritten in the implicit form $\phi\left(s, z_{\mathrm{e}}\right)=0$ and thus one gets

$$
\frac{d z_{\mathrm{e}}}{d s}=-\frac{\frac{\partial \phi}{\partial s}}{\frac{\partial \phi}{\partial z_{\mathrm{e}}}}
$$

Therefore, the slope of the equilibrium Hugoniot curve is specified by equations (30) and (31). On the other hand, the slope of the Rayleigh line (RL) associated to the wave speed $s$ is given by

$$
\left.\frac{d p}{d v}\right|_{\mathrm{RL}}=-\frac{p_{0}}{v_{0}} s^{2}
$$

and, consequentely, the common slope at the tangency point is defined by the condition

$$
-s^{2}=\frac{\frac{\partial g}{\partial s}+\frac{\partial g}{\partial z_{\mathrm{e}}} \frac{d z_{\mathrm{e}}}{d s}}{\frac{\partial f}{\partial s}+\frac{\partial f}{\partial z_{\mathrm{e}}} \frac{d z_{\mathrm{e}}}{d s}}
$$

together with condition (31). Thus, the Chapman-Jouguet velocity $s_{j}$ is obtained as the real solution of equation (33), while the equilibrium concentration at the CJ state follows as the real solution of equation (26) with $s=s_{j}$.

\section{Detonation solution domain}

The steady detonation wave structure can be represented by the Hugoniot diagram which provides a conventional description in the $(v, p)$-plane of the qualitative behaviour of the steady solution, see for example Refs. [18, 19, 25, 20]. In the present study of a steady detonation with reversible reaction, the relevant curves to be represented in the Hugoniot diagram are the Rayleigh lines for $s \geq s_{j}$, the unreacted Hugoniot curve and the equilibrium Hugoniot curve. As previously discussed in the present sub-section, the equilibrium Hugoniot curve is 
defined by its parametric equations involving the equilibrium concentration $z_{\mathrm{e}}$ constrained to the equilibrium condition (26). Therefore, all states of the equilibrium Hugoniot curve must satisfy the condition

$$
p_{\mathrm{e}}=-\frac{\Delta \epsilon}{v_{\mathrm{e}} \ln \left(\frac{1-z_{\mathrm{e}}}{z_{\mathrm{e}}}\right)},
$$

where $z_{\mathrm{e}}$ verifies the condition

$$
1 / 2<z_{\mathrm{e}}<1,
$$

since $p_{\mathrm{e}}>0, v_{\mathrm{e}}>0$, and $\Delta \epsilon>0$ for an exothermic reaction. At the same time, these states also belong to the fixed composition Hugoniot curve (21) for the corresponding concentration $z_{\mathrm{e}}$, and thus

$$
p_{\mathrm{e}}=\frac{2 \mu^{2} z_{\mathrm{e}} \Delta \epsilon+1-\mu^{2} v_{\mathrm{e}}}{v_{\mathrm{e}}-\mu^{2}} .
$$

Combining equations (34) and (36), the following quadratic equation is derived

$$
-\mu^{2} \ln \left(\frac{1-z_{\mathrm{e}}}{z_{\mathrm{e}}}\right) v_{\mathrm{e}}^{2}+\left[\left(1+2 \mu^{2} z_{\mathrm{e}} \Delta \epsilon\right) \ln \left(\frac{1-z_{\mathrm{e}}}{z_{\mathrm{e}}}\right)+\Delta \epsilon\right] v_{\mathrm{e}}-\Delta \epsilon \mu^{2}=0 .
$$

The above equation leads to the unique physically consistent equilibrium state

$$
\begin{aligned}
v_{\mathrm{e}}\left(z_{\mathrm{e}}\right) & =\frac{b-\sqrt{b^{2}-4 a \mu^{2} \Delta \epsilon}}{2 a} \\
p_{\mathrm{e}}\left(z_{\mathrm{e}}\right) & =-\frac{2 \mu^{2} \Delta \epsilon}{b-\sqrt{b^{2}-4 a \mu^{2} \Delta \epsilon}}
\end{aligned}
$$

where

$$
a=a\left(z_{\mathrm{e}}\right)=\mu^{2} \ln \left(\frac{1-z_{\mathrm{e}}}{z_{\mathrm{e}}}\right)
$$

and

$$
b=b\left(z_{\mathrm{e}}\right)=\Delta \epsilon+\left(1+2 \mu^{2} z_{\mathrm{e}} \Delta \epsilon\right) \ln \left(\frac{1-z_{\mathrm{e}}}{z_{\mathrm{e}}}\right) .
$$

Observe that the compatibility condition (35) on $z_{\mathrm{e}}$ induces a limiting condition on the solution domain of the detonation regime. In fact, when $z_{\mathrm{e}} \rightarrow \frac{1}{2}$, equations (34) and (37) determines the limiting conditions as follows

$$
v_{\mathrm{e}} \rightarrow \frac{\gamma-1}{\gamma+1} \quad \text { and } \quad p_{\mathrm{e}} \rightarrow \infty, \quad \text { for } \quad z_{\mathrm{e}} \rightarrow \frac{1}{2}
$$

On the other hand, the solution domain is bounded on the right by the state $\left(v_{\mathrm{r}}, p_{\mathrm{r}}\right)$ with $v_{\mathrm{r}}=1$ and $p_{\mathrm{r}}$ obtained from equation (39), namely

$$
v_{\mathrm{r}}=1 \quad \text { and } \quad p_{\mathrm{r}}=1+(\gamma-1) z_{\mathrm{e}} \Delta \epsilon .
$$

In conclusion, the solution domain of the detonation regime is defined by the conditions

$$
\frac{\gamma-1}{\gamma+1}<v \leq 1, \quad \infty>p \geq 1+(\gamma-1) z_{\mathrm{e}} \Delta \epsilon,
$$

where $z_{\mathrm{e}}$ is such that $v_{\mathrm{e}}\left(z_{\mathrm{e}}\right)=1$. 


\section{Detonation wave solution}

In the present analysis only wave solutions with final state in the strong detonation branch are considered. In this case, the speed of the detonation wave $s$ satisfies the condition $s \geq s_{j}$, with $s_{j}$ being the CJ velocity previously defined.

\section{Strong detonation solution}

A steady detonation wave solution consists of a non-reactive shock wave connected to a continuous reacting flow. The wave structure is characterized by solving two problems. The first is an algebraic problem leading to the post-shock state as the solution of the proper jump RH conditions. The second is an initial value problem associated to the steady reactive Euler equations with initial conditions assigned at the post-shock state. The latter problem describes the continuous reacting flow behind the shock wave.

i) post-shock state

The progress variable $z$ does not change through a non-reactive shock, so that the rate equation results to be conservative and a further $\mathrm{RH}$ condition is obtained, namely

$$
z_{*}=z_{0}=0 .
$$

The post-shock state is then characterized by the RH jump conditions (17-19) enforced with condition (45). Two solutions are obtained, namely the so-called ambient solution

$$
p_{*}=\frac{p}{p_{0}}=1, \quad v_{*}=\frac{v}{v_{0}}=1, \quad w_{*}=\frac{w}{\sqrt{p_{0} v_{0}}}=s
$$

and the post-shock solution

$$
p_{*}=\frac{p}{p_{0}}=\frac{2}{\gamma+1} s^{2}-\frac{\gamma-1}{\gamma+1}, \quad v_{*}=\frac{v}{v_{0}}=\frac{\gamma-1}{\gamma+1}+\frac{2}{s^{2}} \frac{\gamma}{\gamma+1}, \quad w_{*}=\frac{w}{\sqrt{p_{0} v_{0}}}=v_{*} s .
$$

For a given propagation velocity $s$ of the shock wave, expressions (47) characterize the post-shock state just behind the wave front. In particular, when $s \gg 1$, the post-shock solution tends to the so-called strong shock limit, characterized by

$$
p_{*}=\frac{p}{p_{0}}=\frac{2}{\gamma+1} s^{2}, \quad v_{*}=\frac{v}{v_{0}}=\frac{\gamma-1}{\gamma+1} \quad \text { and } \quad w_{*}=\frac{w}{\sqrt{p_{0} v_{0}}}=\frac{\gamma-1}{\gamma+1} s .
$$

In this strong shock limit, the flow velocity $u=s-w$ in the laboratory frame reads

$$
\frac{u}{\sqrt{p_{0} v_{0}}}=\frac{2}{\gamma+1} s
$$

\section{ii) Continuos reacting flow}

The evolution of the mixture through the shock front raises pressure and temperature to very high values, so that an exothermic chemical reaction initiates at the post-shock state and evolves in the reaction zone attached to the shock wave. The continuous 
flow within this zone is determined by solving (for $\xi>0$ ) the dimensionless system of differential equations

$$
\begin{aligned}
& \frac{d p}{d \xi}=\frac{p}{T} w \frac{(\gamma-1) \Delta \epsilon}{w^{2}-\gamma T} r, \\
& \frac{d T}{d \xi}=\left(w-\frac{T}{w}\right) \frac{(\gamma-1) \Delta \epsilon}{w^{2}-\gamma T} r, \\
& \frac{d w}{d \xi}=-\frac{(\gamma-1) \Delta \epsilon}{w^{2}-\gamma T} r, \\
& \frac{d z}{d \xi}=\frac{r}{w},
\end{aligned}
$$

which follows from equations (1-4) re-written in terms of the steady variable $\xi$ defined by expression (15). In the above equations, $p, T, w, \Delta \epsilon, \xi$ and $r$ are given, respectively, in units of $p_{0}, T_{0}, \sqrt{p_{0} v_{0}}, p_{0} v_{0}, \tau_{0} \sqrt{p_{0} v_{0}}$ and $\tau_{0}^{-1}$, where

$$
\tau_{0}=\frac{1}{2 n_{0} a^{2}} \sqrt{\frac{m}{\pi k T_{0}}}
$$

is a reference time of order of the collision mean free path. In view of the application performed in Section 5, the temperature $T$ has been considered among the main fields, instead of the mixture mass density $\rho$. Moreover, the dimensionless form of the reaction rate $r$ reads

$$
r=\frac{p}{\sqrt{T}} \exp \left(-\frac{\epsilon_{f}}{T}\right)\left[(1-z)^{2}-z^{2} \exp \left(-2 \frac{\Delta \epsilon}{T}\right)\right],
$$

with $\epsilon_{f}$ given in units of $k T_{0}$. The initial conditions at $\xi=0$ for the system of equations (50-53) are provided by the post-shock expressions (47).

\section{Compatibility detonation conditions}

The existence of the detonation solution depends on the compatibility of the non-reactive shock wave with the continuous reactive flow. Therefore, the Lax conditions on the characteristic velocities of the shock wave, namely the conditions $s>u+c$ at the initial state, and $u<s<u+c$ at the post-shock state (see [26]) are combined with the detonation condition on the characteristic velocities of the reactive Euler equations, namely $u<s<u+c$ at the reaction zone (see $[5,19,25])$. Therefore, the compatibility conditions which must be verified by the detonation solution are

$$
s>u+c \text { for } \xi<0 \text { and } u<s<u+c \text { for } \xi \geq 0 .
$$

\section{Solution procedure}

In order to determine the steady detonation wave solution, the algebraic problem of evaluating the post-shock state, and the initial value problem of characterizing the continuous reacting flow are solved for assigned initial state of the reactive flow. A suitable input parameter space is considered, including $\gamma, \Delta \epsilon, \epsilon_{f}$, and

$$
f=\left(s / s_{j}\right)^{2} \geq 1
$$

which specifies the overdrive degree of the detonation wave. The steady solution is determined applying the solution procedure established on the following steps: 
1) CJ velocity. For the considered input parameters $\gamma$ and $\Delta \epsilon$, determine the wave velocity $s_{j}$ and the equilibrium concentration $z_{\mathrm{e}}$ at the CJ state by solving equations (26) and (33).

2) Detonation velocity. For the considered overdrive degree $f$, use the value of $s_{j}$ obtained in the first step to evaluate the detonation velocity $s=s_{j} \sqrt{f}$.

3) Post-shock state. For the detonation velocity $s$ determined in the second step, evaluate the post-shock state - say $v_{*}, p_{*}, w_{*}$ - by using (47).

4) Continuous reacting flow. For the input parameters $\gamma, \Delta \epsilon$ and $\epsilon_{f}$, characterize the continuous reacting flow in the reaction zone, solving the system of differential equations (50-53), with the reaction rate $r$ specified by (55), and with initial conditions at $\xi=0$ specified by the post-shock state evaluated in the third step.

\section{$5 \quad$ Numerical analysis}

The analysis developed in the previous section is here applied to solve numerically the detonation problem. The solution procedure is applied and the compatibility detonation conditions (56) are numerically verified. The solution of the numerical problem provides, first, the Chapman-Jouguet velocity $s_{j}$, then, for each $s \geq s_{j}$, the corresponding post-shock state, and, finally, the continuous reacting flow in the reaction zone. The computation of $s_{j}$ depends on $\gamma$ and $\Delta \epsilon$, only. The evaluation of the post-shock state depends on $\gamma, \Delta \epsilon$ and $f$. The configuration of the continuous reacting flow depends, in turn, on $\gamma, \Delta \epsilon, f$ and $\epsilon_{f}$. In particular, the equilibrium final state can be obtained independently of the continuous reacting flow, requiring the parameters $\gamma, \Delta \epsilon$ and $f$, only.

The numerical computations performed in this section are addressed to provide the structure of the detonation solution in the $(v, p)$-plane, and to study the influence of the overdrive degree $f$ and forward activation energy $\epsilon_{f}$ on the wave profiles of the steady solution.

Input data

The input data for the numerical application are the dimensionless pre-shock state of the reactive flow

$$
\frac{p}{p_{0}}=1, \quad \frac{v}{v_{0}}=1, \quad \frac{u}{\sqrt{p_{0} v_{0}}}=0 \quad \text { and } \quad z=0,
$$

and the parameters

$$
\gamma=\frac{5}{3} \quad \text { and } \quad \Delta \epsilon=1.0
$$

which are held fixed.

Chapman-Jouguet state

For the material properties of the reactive mixture specified by equation (59), the ChapmanJouguet velocity $s_{j}$, the corresponding equilibrium concentration $z_{\mathrm{e}}$ and the state variables at the CJ state are

$$
s_{j}=2.25181, \quad z_{\mathrm{e}}=0.64269, \quad v_{\mathrm{e}} / v_{0}=0.748259 \quad \text { and } \quad p_{\mathrm{e}} / p_{0}=2.27649 \text {. }
$$




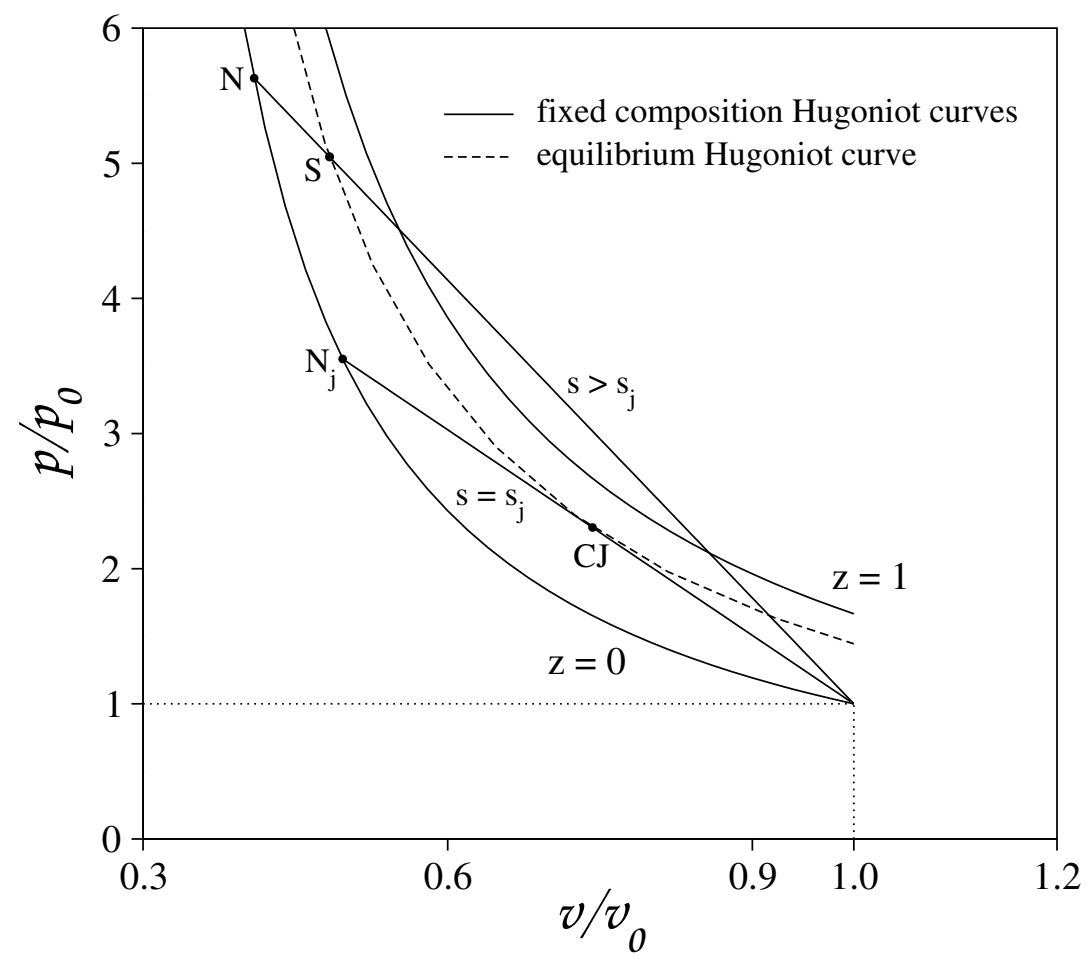

Figure 1: Hugoniot diagram for $\gamma=5 / 3$ and $\Delta \epsilon=1.0$. Rayleigh lines for $s_{j}=2.25181$ and $s=2.8$, unreacted and complete reaction Hugoniot curves and equilibrium Hugoniot curve (dashed line). Segments $\mathrm{N}_{j} \mathrm{CJ}$ and NS indicate the solution for $s=s_{j}$ and $s>s_{j}$, respectively.

Wave structure in the $(v, p)$-plane

The structure of the steady detonation solution is represented in the Hugoniot diagram of Fig. 1. Two Rayleigh lines are drawn for $s=s_{j}=2.25181$ and $s=2.8$. The diagram shows the unreacted Hugoniot curve, locus of the initial state and all post-shock states $N$ obtained for different values of $s \geq s_{j}$. The complete reaction Hugoniot curve is drawn for sake of completeness, even though the solution in the present case of reversible reaction does not match this curve. The equilibrium Hugoniot curve, locus of all equilibrium final states, is the dashed curve represented in the diagram. This curve is drawn interpolating a large set of equilibrium final states reached for assigned values of $s$ in the range $[2.25181 ; 6.00]$. In particular, Table 1 shows the states on the strong branch of the equilibrium Hugoniot curve. The equilibrium final state on the lowest point of the curve is determined by expressions (43), resulting

$$
\left(v_{\mathrm{r}}, p_{\mathrm{r}}\right)=(1,1.44433) \quad \text { and } \quad z_{\mathrm{r}}=0.666492 .
$$

For each value of $s \geq s_{j}$, the complete strong detonation solution is represented in the diagram by the segment $N S$ determined by the intersections of the corresponding Rayleigh line with the unreacted and equilibrium Hugoniot curves, respectively. 


\begin{tabular}{|c|c|c|c|}
\hline$s$ & $z_{\mathrm{e}}$ & $v / v_{0}$ & $p / p_{0}$ \\
\hline \hline 2.25181 & 0.64269 & 0.74826 & 2.27649 \\
\hline 2.26 & 0.63829 & 0.71077 & 2.47730 \\
\hline 2.28 & 0.63483 & 0.68320 & 2.64687 \\
\hline 2.30 & 0.63235 & 0.66438 & 2.77544 \\
\hline 2.32 & 0.63026 & 0.64908 & 2.88880 \\
\hline 2.40 & 0.62354 & 0.60354 & 3.28362 \\
\hline 2.50 & 0.61681 & 0.56289 & 3.73194 \\
\hline 2.60 & 0.61100 & 0.53134 & 4.16814 \\
\hline 2.80 & 0.60099 & 0.48367 & 5.04802 \\
\hline 3.00 & 0.59239 & 0.44857 & 5.96286 \\
\hline 3.20 & 0.58484 & 0.42143 & 6.92458 \\
\hline 4.00 & 0.56186 & 0.35536 & 11.31420 \\
\hline 5.00 & 0.54361 & 0.31589 & 18.10270 \\
\hline 6.00 & 0.53207 & 0.29521 & 26.37250 \\
\hline
\end{tabular}

Table 1: Equilibrium final states in the strong branch of the equilibrium Hugoniot curve.

Profiles of the steady solution

The parameters $f$ and $\epsilon_{f}$ have a significant role in the detonation structure and their influence on the steady solution is treated separately in two different study cases.

In each case, the privileged parameter varies in a suitable range and all the other parameters are held fixed. Some representative detonation profiles are drawn in Figs. 2 and 3, where the steady variable $\xi$ is given in a logarithmic scale and the state variables $p, T$ and $w$ are given in units of post-shock values $p_{*}, T_{*}$ and $w_{*}$, respectively. The profiles show the behaviour of the state variables from the post-shock state attached to the shock front, $\xi=0$ until the same distance from the shock, $\xi=100$. The equilibrium of the state variables is reached when all profiles become flat.

Case 1 - Influence of $f$ on the steady solution

Wave structure for varying overdrive degree $f$. In this case, the properties of the mixture are specified by the data (59) and by

$$
\epsilon_{f}=2.0
$$

The privileged parameter takes the values

$$
f=1, \quad f=1.5, \quad f=3 .
$$

For these values of $f$, the detonation velocity and the post-shock state - starred quantities - are shown in Table 2. The wave structure for the Eulerian reacting gas mixture is represented in Fig. 2 in terms of the pressure, temperature, waveframe velocity and progress variable profiles for $f=1$ (solid lines), $f=1.5$ (dashed lines) and $f=3.0$ (dot-dashed lines). For each value of $f$, all profiles match their equilibrium values for the same distance $\xi$ from the shock. With an accuracy of order $10^{-4}$, the equilibrium is reached when $\xi=40$ for $f=1, \xi=6$ for $f=1.5$ and $\xi=2$ for $f=3.0$. 

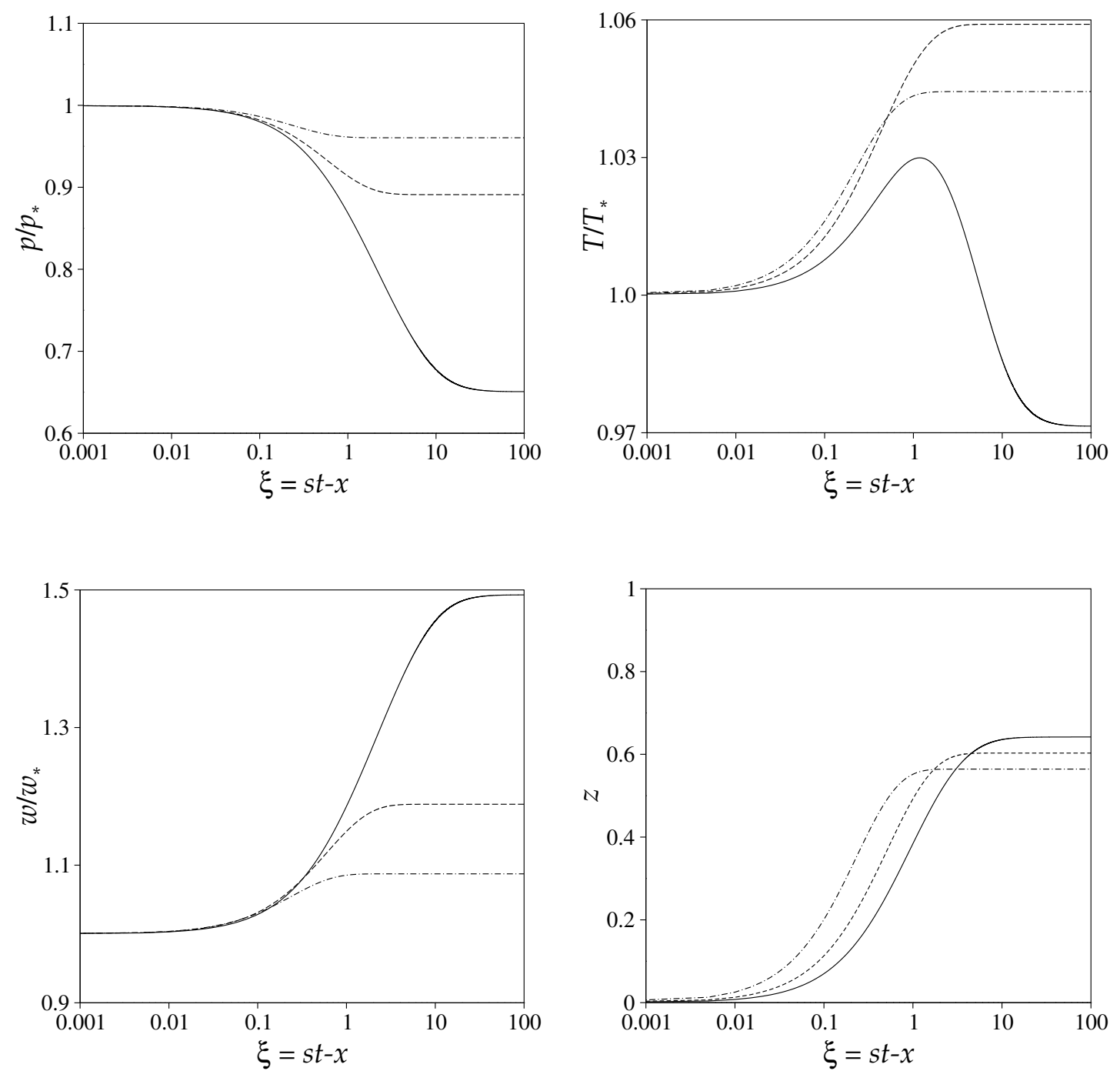

Figure 2: Wave structure in Case 1. Pressure, temperature, waveframe velocity and progress variable profiles for $f=1.0$ (solid line), $f=1.5$ (dashed line) and $f=3.0$ (dot-dashed line). The input parameters are $\gamma=5 / 3, \Delta \epsilon=1.0$ and $\epsilon_{f}=2.0$.

The pressure profiles show a typical reacting flow in the reaction zone characterized by a rarefaction until the pressure becomes constant and the equilibrium is reached. Moreover, the pressure variation in the reaction zone is more pronounced for smaller values of $f$. This behaviour is in agreement with the Hugoniot diagram of Fig. 1 where one can recognize that increasing values of $f$ lead to higher post-shock states $N$ with greater pressure. Consequently, since $p$ is expressed in units of the pressure $p^{*}$ at the state $N$, the pressure profiles in Fig. 2 show a smaller variation across the reaction zone for greater values of $f$.

The temperature profiles indicate that the temperature sensitivity of the reaction rate is weaker for stronger overdrive degrees. The profiles of $T$ for $f=1.5$ and $f=3.0$ show a typical behavior., whereas the profile for $f=1$ exhibits a peak before the equilibrium. This 


\begin{tabular}{|c|c|c|c|c|c|c|}
\hline$f$ & $s$ & $\rho_{*}$ & $p_{*}$ & $w_{*}$ & $z_{*}$ & $T_{*}=p_{*} / \rho_{*}$ \\
\hline \hline 1.0 & 2.25181 & 2.0138 & 3.5521 & 1.1181 & 0 & 1.7639 \\
\hline 1.5 & 2.75789 & 2.4132 & 5.4532 & 1.1427 & 0 & 2.2597 \\
\hline 3.0 & 3.90025 & 3.0102 & 11.156 & 1.2955 & 0 & 3.7061 \\
\hline
\end{tabular}

Table 2: Post-shock states for three values of the overdrive degree.

abrupt shape can be justified by the reversibility of the chemical process and the endothermic character of the backward reaction. In fact, when $f=1$ and the distance $\xi$ from the shock wave is approximately 1.5, the backward reaction becomes dominant and the temperature strongly decreases. When $f=1.5$ and $f=3.0$, the profiles of $T$ do not share such aspect since the exothermic forward reaction is prevalent. A further aspect is observed when $\xi$ is approximately 0.7. In detail, until such distance is reached, increasing values of $f$ imply larger temperature variation, and for a greater distance, $\xi>0.7$, the profiles for $f=1.5$ and $f=3.0$ exchange their relative setting and show the expected tendency to the equilibrium. The behaviour of $T$ for $\xi<0.7$ can be justified by the fact that an increase of the thermal energy typically occurs when the detonation velocity becomes greater, however these effects are recognizable only in the neighbourhood of the shock wave.

The profiles of the waveframe velocity show an increasing behaviour in the reaction zone until the equilibrium state is reached, which is more pronounced for smaller values of $f$.

Finally, the profiles of the progress variable $z$ show a common pattern and illustrate that the variation in the product concentration is greater for smaller values of $f$.

Case 2 - Influence of $\epsilon_{f}$ on the steady solution.

Wave structure for varying forward activation energy $\epsilon_{f}$. The input properties of the mixture are specified by the data (59) and the detonation overdrive degree is fixed as

$$
f=1.5 \text {. }
$$

The detonation velocity and the post-shock state are then given by

$$
s=2.75789, \quad \rho^{*}=2.4132, \quad p^{*}=5.4532, \quad w^{*}=1.1427 .
$$

In this case, the privileged parameter takes the values

$$
\epsilon_{f}=2.0, \quad \epsilon_{f}=10.0 \quad \text { and } \quad \epsilon_{f}=30.0 .
$$

The wave structure is represented in Fig. 3 for $\epsilon_{f}=2.0$ (solid lines), $\epsilon_{f}=10.0$ (dashed lines) and $\epsilon_{f}=30.0$ (dot-dashed lines). The profiles corresponding to $\epsilon_{f}=30.0$ represent a limiting case in which the state variables remain constant in all states behind the shock front. Hence the detonation wave solution approaches the step shock structure of an inert Eulerian gas mixture. On the other hand, the profiles for $\epsilon_{f}=2.0$ and $\epsilon_{f}=10.0$ represent the continuous reacting flow with the particularity that the final state is characterized by the same values of $p, T, w$ and $z$ for both $\epsilon_{f}=2.0$ and $\epsilon_{f}=10.0$. With an accuracy of order $10^{-4}$, all 

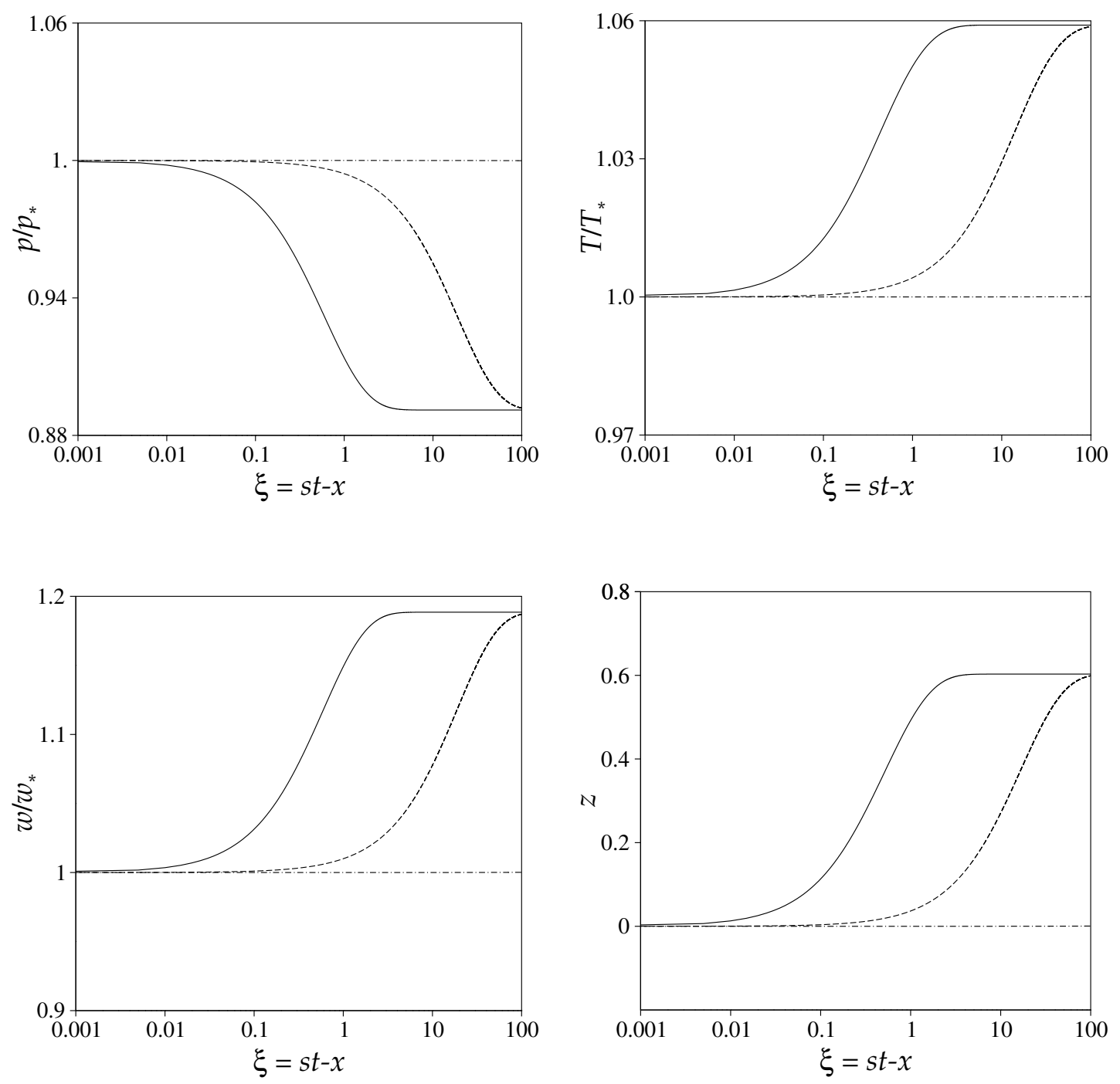

Figure 3: Wave structure in Case 2. Pressure, temperature, waveframe velocity and progress variable profiles for $\epsilon_{f}=2.0$ (solid line), $\epsilon_{f}=10.0$ (dashed line) and $\epsilon_{f}=30.0$ (dot-dashed line). The input parameters are $\gamma=5 / 3, \Delta \epsilon=1.0$ and $f=1.5$.

profiles match their equilibrium values for the same distance from the shock, namely $\xi=5$ for $\epsilon_{f}=2.0$ and $\xi=100$ for $\epsilon_{f}=10.0$,

The activation energy influences the detonation wave structure at level of the reaction zone only, but does neither affect the post-shock state $N$ nor the equilibrium final state, as expected. In fact, it is enough to recall that the state $N$ is defined by Eq. (47) which does not include $\epsilon_{f}$, and that the equilibrium final state corresponds to the vanishing of the square bracket in Eq. (55), independently of $\epsilon_{f}$.

Moreover, the limiting case corresponding to $\epsilon_{f}=30.0$ reveals that the state variables do not vary behind the post-shock state $N$. The continuous reacting flow exhibits an uniform zone 
of constant states, which represents the induction zone typical of the square-wave detonation obtained for very high activation energy $[1,18,27]$. Such behaviour is justified by the contribution of $\epsilon_{f}$ to the pre-exponential factor of the reaction rate $r$, implying that $r$ becomes negligible for higher values of $\epsilon_{f}$, see Eq. (55). This analytical result is expected since the activation energy represents the energy barrier that the gas particles must overcome in order to interact with chemical reaction. Therefore, for high values of $\epsilon_{f}$, only few particles go beyond this energy barrier and the chemical reaction becomes negligible.

The profiles of the state variables for $\epsilon_{f}=2.0$ and $\epsilon_{f}=10.0$ show a typical continuous reacting flow. The final state is characterized by the same values of $p, w, T$ and $z$ for both $\epsilon_{f}=2.0$ and $\epsilon_{f}=10.0$. These profiles exhibit a constant branch in the neighbourhood of the post-shock state which is longer for $\epsilon_{f}=10.0$. This behaviour is justified by the fact that a higher value of the activation energy delays the onset of the chemical reaction. A further interesting effect of the activation energy on the wave structure consists in the enlarging of the reaction zone for greater values of $\epsilon_{f}$. In fact, the profiles show that the equilibrium state is reached for $\xi=5$ when $\epsilon_{f}=2.0$ and for $\xi=100$ when $\epsilon_{f}=10.0$.

\section{Formulation of the linear stability problem}

In this section a normal mode approach to the stability problem for the steady detonation wave structure identified in Section 5 is studied in presence of two dimensional disturbances. The detonation stability is classically studied (see, for instance, Refs. [28, 29, 30, 31, 32]), assuming that a small rear boundary disturbance is instantaneously assigned and induces a distortion on the shock wave location, giving rise to small state variables perturbations which propagate in the reaction zone. Therefore the shock distortion affects the steady state variables so that reaction zone does not admit anymore a steady configuration. The stability problem consists then in studying the evolution of the state variables perturbations, from the post-shock state until the equilibrium final state.

The mathematical formulation of stability problem with two dimensional disturbances starts from the bidimensional reactive Euler equations,

$$
\begin{aligned}
& \frac{\partial v}{\partial t}+u_{i} \frac{\partial v}{\partial x_{i}}-v \frac{\partial u_{i}}{\partial x_{i}}=0, \\
& \frac{\partial u_{j}}{\partial t}+u_{i} \frac{\partial u_{j}}{\partial x_{i}}+v \frac{\partial p}{\partial x_{i}}=0, \quad j=1,2 \\
& \frac{\partial p}{\partial t}+u_{i} \frac{\partial p}{\partial x_{i}}+\rho c^{2} \frac{\partial u_{i}}{\partial x_{i}}=\rho c^{2} \sigma r \\
& \frac{\partial z}{\partial t}+u_{i} \frac{\partial z}{\partial x_{i}}=r
\end{aligned}
$$

where $u_{1}$ and $u_{2}$ are the components of the flow velocity in the $x$ and $y$ directions, respectively. The formulation proceeds by transforming to the shock-attached coordinate system

$$
\xi=s t-x+\psi(y, t),
$$

where $\psi(y, t)$ represents the perturbation to the steady shock location due to a small transverse perturbation. Since linear stability requires to seek solutions of the transformed reactive 
Euler equations which deviate by a small amount from the known steady state solution, a normal mode expansion about the steady state can be assumed in the form

$$
\boldsymbol{q}(\xi, y, t)=\boldsymbol{q}^{*}(\xi)+\boldsymbol{q}^{\prime}(\xi) e^{\alpha t+i k y} \quad \text { and } \quad \psi(\xi, y, t)=\psi^{\prime}(\xi) e^{\alpha t+i k y}
$$

where

$$
\boldsymbol{q}=\left(\begin{array}{c}
v \\
s-u_{1} \\
u_{2} \\
p \\
z
\end{array}\right)=\left(\begin{array}{c}
v \\
w \\
u_{2} \\
p \\
z
\end{array}\right), \quad \boldsymbol{q}^{*}=\left(\begin{array}{c}
v^{*} \\
w^{*} \\
0 \\
p^{*} \\
z^{*}
\end{array}\right) \quad \text { and } \quad \boldsymbol{q}^{\prime}=\left(\begin{array}{c}
v^{\prime} \\
w^{\prime} \\
u_{2}^{\prime} \\
p^{\prime} \\
z^{\prime}
\end{array}\right)
$$

represent the state vector, the steady-state vector and the perturbation amplitude vector, respectively. Furthermore, $\operatorname{Re}(\alpha)$ is the disturbance growth rate, $\operatorname{Im}(\alpha)$ the disturbance frequency, $k$ the disturbance wavenumber, and $\psi^{\prime}$ the spatial perturbation amplitude of the shock location.

\section{Stability equations}

The reactive Euler equations, referred to the coordinate frame (69), and linearized through expansions (70), can be written in the following dimensionless matrix form in the unknown perturbation amplitudes $\boldsymbol{q}^{\prime}$

$$
\mathcal{A} \cdot \frac{d \boldsymbol{q}^{\prime}}{d \xi}+\mathcal{B} \cdot \boldsymbol{q}^{\prime}+\mathcal{C} \psi^{\prime}=0
$$

where

$$
\begin{aligned}
& \mathcal{A}=\left(\begin{array}{ccccc}
w^{*} & -v^{*} & 0 & 0 & 0 \\
0 & w^{*} & 0 & v^{*} & 0 \\
0 & 0 & w^{*} & 0 & 0 \\
0 & \gamma p^{*} & 0 & w^{*} & 0 \\
0 & 0 & 0 & 0 & w^{*}
\end{array}\right), \quad \mathcal{C}=\left(\begin{array}{c}
\alpha \frac{d v^{*}}{d \xi} \\
\alpha \frac{d w^{*}}{d \xi} \\
i k v^{*} \frac{d p^{*}}{d \xi} \\
\alpha \frac{d p^{*}}{d \xi} \\
\alpha \frac{d z^{*}}{d \xi}
\end{array}\right), \\
& \mathcal{B}=\left(\begin{array}{ccccc}
\alpha-\frac{d w^{*}}{d \xi} & \frac{d v^{*}}{d \xi} & -i k v^{*} & 0 & 0 \\
\frac{d p^{*}}{d \xi} & \alpha+\frac{d w^{*}}{d \xi} & 0 & 0 & 0 \\
0 & 0 & \alpha & i k v^{*} & 0 \\
\frac{(1-\gamma) \Delta \epsilon}{v^{*}}\left(r_{v}^{*}-\frac{r^{*}}{v^{*}}\right) & \frac{d p^{*}}{d \xi} & i k \gamma p^{*} & \alpha+\gamma \frac{d w^{*}}{d \xi}+\frac{(1-\gamma) \Delta \epsilon}{v^{*}} r_{p}^{*} & \frac{(1-\gamma) \Delta \epsilon}{v^{*}} r_{z}^{*} \\
-r_{v}^{*} & \frac{d z^{*}}{d \xi} & 0 & -r_{p}^{*} & \alpha-r_{z}^{*}
\end{array}\right) .
\end{aligned}
$$


In the above expressions, $\alpha$ is given in units of $\tau_{0}^{-1}, k$ in units of $\left(s \tau_{0}\right)^{-1}$ and $\xi$ in units of $s \tau_{0}$, where $\tau_{0}$ is the reference time defined by expression (54). Moreover, the reaction rate $r(v, p, z)$ has been linearized in the form

$$
r=r^{*}+r_{v}^{*}\left(v-v^{*}\right)+r_{p}^{*}\left(p-p^{*}\right)+r_{z}^{*}\left(z-z^{*}\right),
$$

where $r_{v}^{*}, r_{p}^{*}$ and $r_{z}^{*}$ denote the partial derivatives of $r$.

The ordinary differential equations (72) constitute the stability equations for the present modeling.

\section{Boundary conditions}

The boundary conditions to be joined to the stability equations (72) are derived from the bidimensional shock relations at $\xi=0$. The first step is to refer such relations to the unsteady shock described at all times by the curve

$$
f(x, y, t)=x-s t-\psi(y, t)=0,
$$

whose unit normal $\boldsymbol{n}$ and tangent unit vector $\boldsymbol{t}$ are

$$
\boldsymbol{n}=\frac{\boldsymbol{i}-i k \psi \boldsymbol{j}}{\sqrt{1-k^{2} \psi^{2}}}, \quad \boldsymbol{t}=\frac{i k \psi \boldsymbol{i}+\boldsymbol{j}}{\sqrt{1-k^{2} \psi^{2}}} .
$$

The normal shock velocity in the unsteady shock-attached frame (69) is given by

$$
\boldsymbol{s}=\left(s+\frac{\partial \psi}{\partial t}\right) \boldsymbol{n}=(s+\alpha \psi) \boldsymbol{n}
$$

The resulting conditions on the unsteady shock curve read

$$
\begin{aligned}
& {\left[\rho\left(u_{i}-s_{i}\right) n_{i}\right]=0,} \\
& {\left[\rho u_{j} n_{j}\left(u_{i}-s_{i}\right) n_{i}+p\right]=0,} \\
& {\left[\rho e\left(u_{i}-s_{i}\right) n_{i}+p u_{i} n_{i}\right]=0,} \\
& {\left[u_{i} t_{i}\right]=0,} \\
& {[z]=0,}
\end{aligned}
$$

where the index $i$ indicates $x$ and $y$ components, and the square brackets denote the difference between the quantities inside the brackets evaluated ahead and behind the shock surface.

Linearization of $\boldsymbol{n}, \boldsymbol{t}$ and $\boldsymbol{s}$ with respect to the perturbations leads to the

$$
\boldsymbol{n}=\boldsymbol{i}-i k \psi \boldsymbol{j}, \quad \boldsymbol{t}=i k \psi \boldsymbol{i}+\boldsymbol{j}, \quad \boldsymbol{s}=\boldsymbol{i}-i k s \psi \boldsymbol{j},
$$

so that linearization of conditions (77-81) through the normal mode expansions (70) leads to boundary conditions 


$$
\begin{gathered}
v^{\prime}=-\frac{4 \gamma}{s^{3}(\gamma+1)} \alpha \psi^{\prime} \\
w^{\prime}=-\frac{2}{\gamma+1} \frac{s^{2}+\gamma}{s^{2}} \alpha \psi^{\prime} \\
u_{2}^{\prime}=-i k \frac{2}{\gamma+1} \frac{s^{2}-\gamma}{s} \alpha \psi^{\prime} \\
p^{\prime}=\frac{4 s}{\gamma+1} \alpha \psi^{\prime} \\
z^{\prime}=0
\end{gathered}
$$

Accoustic modes at the end of the reaction zone

At the end of the reaction zone, the steady-state variables reach the equilibrium and then remain constant. Thus, all steady-state $\xi$-gradients vanish so that $\mathcal{C}=0$ in Eq. (72). The stability equations reduce to

$$
\mathcal{A} \cdot \frac{d \boldsymbol{q}^{\prime}}{d \xi}+\mathcal{B} \cdot \boldsymbol{q}^{\prime}=0
$$

where the matrix $\mathcal{B}$ simplifies to

$$
\mathcal{B}=\left(\begin{array}{ccccc}
\alpha & 0 & -i k v^{*} & 0 & 0 \\
0 & \alpha & 0 & 0 & 0 \\
0 & 0 & \alpha & i k v^{*} & 0 \\
\frac{(1-\gamma) \Delta \epsilon}{v^{*}} r_{v}^{*} & 0 & i k \gamma p^{*} & \alpha+\frac{(1-\gamma) \Delta \epsilon}{v^{*}} r_{p}^{*} & \frac{(1-\gamma) \Delta \epsilon}{v^{*}} r_{z}^{*} \\
-r_{v}^{*} & 0 & 0 & -r_{p}^{*} & \alpha-r_{z}^{*}
\end{array}\right)
$$

In view of a perturbation wave analysis, the $\xi$-spatial dependence of the amplitudes $\boldsymbol{q}^{\prime}$ is represented as a Fourier mode in terms of the wavenumber $\eta$, i.e.,

$$
\boldsymbol{q}^{\prime}=\overline{\boldsymbol{q}} e^{i \eta \xi}
$$

Consequently, the differential system (88) reduces to the algebraic homogeneous system

$$
(i \eta \mathcal{A}+\mathcal{B}) \cdot \overline{\boldsymbol{q}}=0
$$

for which the dispersion relation reads

$$
\begin{aligned}
\left(\alpha+i \eta w^{*}\right)^{2}\left\{\left(\alpha+i \eta w^{*}\right)^{3}-\left(\alpha+i \eta w^{*}\right)^{2}\left(r_{z}^{*}+(\gamma-1) \Delta \epsilon \frac{r_{p}^{*}}{v^{*}}\right)\right. & \\
& \left.+\left(\alpha+i \eta w^{*}\right) c_{*}^{2}\left(\eta^{2}+k^{2}\right)-c_{*}^{2}\left(\eta^{2}+k^{2}\right)\left(r_{z}^{*}+\frac{(\gamma-1)}{\gamma} \Delta \epsilon \frac{r_{v}^{*}}{p^{*}}\right)\right\}=0 .
\end{aligned}
$$


For fixed growth rate $\alpha \in \mathbb{C}$, the dispersion relation (92) can be used to determine the five propagation modes corresponding to the roots $\eta$ of Eq. (92). Taking into account that the chemical reaction rate of the present detonation modeling is such that $r_{v}^{*} \approx \gamma\left(p^{*} / v^{*}\right) r_{p}^{*}$ with an accuracy of $10^{-3}$, the dispersion relation (92) reduces to

$$
\left(\alpha+i \eta w^{*}\right)^{2}\left(\left(\alpha+i \eta w^{*}\right)^{2}+c^{* 2}\left(\eta^{2}+k^{2}\right)\right)\left(\alpha+i \eta w^{*}-b\right)=0
$$

where

$$
b=r_{z}^{*}+\frac{\gamma-1}{\gamma} \Delta \epsilon \frac{r_{v}^{*}}{p^{*}}
$$

Solving the above dispersion relation, the roots are

$$
\begin{gathered}
\eta_{1,2}=i \frac{\alpha}{w^{*}}, \\
\eta_{3,4}=\frac{-i \alpha w^{*} \pm i c^{*} \sqrt{\alpha^{2}+k^{2}\left(c^{* 2}-w^{* 2}\right)}}{c^{* 2}-w^{* 2}} \\
\eta_{5}=i \frac{\alpha-b}{w^{*}} .
\end{gathered}
$$

The roots $\eta_{1,2}$ determine vorticity and entropic waves and the roots $\eta_{3,4}$ define backward and forward acoustic waves. The expressions (95) and (96) recover those obtained in other papers (see, for instance $[31,33]$ ). The root $\eta_{5}$ is related to the chemical reaction and its expression shows an explicit dependence on the derivatives of the reaction rate. Such root reduces to the one of paper [31] in the particular case in which $r_{v}^{*}=r_{p}^{*}=0$ at the end of the reaction zone. These roots define five independent eigenvectors,

$$
\boldsymbol{r}_{1}=\left(\begin{array}{c}
1 \\
0 \\
0 \\
0 \\
-r_{v}^{*} \\
r_{z}^{*}
\end{array}\right), \boldsymbol{r}_{2}=\left(\begin{array}{c}
0 \\
1 \\
\frac{-\eta_{2}}{k} \\
0 \\
0
\end{array}\right), \boldsymbol{r}_{3}=\left(\begin{array}{c}
-\frac{v^{* 2}}{c^{* 2}} \\
\frac{-i \eta_{3} v^{*}}{\alpha+i \eta_{3} w^{*}} \\
\frac{-i k v^{*}}{\alpha+i \eta_{3} w^{*}} \\
1 \\
0
\end{array}\right), \boldsymbol{r}_{4}=\left(\begin{array}{c}
-\frac{v^{* 2}}{c^{* 2}} \\
\frac{-i \eta_{4} v^{*}}{\alpha+i \eta_{4} w^{*}} \\
\frac{-i k v^{*}}{\alpha+i \eta_{4} w^{*}} \\
1 \\
0
\end{array}\right), \boldsymbol{r}_{5}=\left(\begin{array}{c}
\left(\eta_{5}^{2}+k^{2}\right) \frac{v^{* 2}}{b^{2}} \\
-i \eta_{5} \frac{v^{*}}{b} \\
-i k \frac{v^{*}}{b} \\
1 \\
\frac{c^{* 2}\left(\eta_{5}^{2}+k^{2}\right)+b^{2}}{(\gamma-1) \Delta \epsilon} \frac{v^{*}}{b^{2}}
\end{array}\right),
$$

so that any disturbance $\boldsymbol{q}^{\prime}$ can be expressed as linear combination of these eigenvectors,

$$
\boldsymbol{q}^{\prime}=\sum_{i=1}^{5} c_{i} \boldsymbol{r}_{i}
$$

where $c_{i}, i=1, \ldots, 5$, are the coefficients of the combination. Such coefficients can be determined solving the equation

$$
c=\mathcal{R}^{-1} \boldsymbol{q}^{\prime}
$$

where $\mathcal{R}$ is the matrix whose columns are the eigenvectors and $\boldsymbol{c}$ the vector of the coefficients. The detonation stability essentially requires that the disturbance induced on the 
shock location is the unique perturbation source of the instabilities in the reaction zone, see, for example, $[28,31,34]$. Thus, the instabilities $\boldsymbol{q}^{\prime}$ can not depend on the forward acoustic wave $\boldsymbol{r}_{4}$ traveling from the rear boundary towards the shock front, and the corresponding coefficient $c_{4}$ in the linear combination (98) must vanish. From Eq. (99), one obtains the radiation condition of the present modeling in the form

$$
\begin{gathered}
i \frac{\left(b-r_{z}\right)}{v^{*}} \frac{\alpha^{2}-k^{2} w^{* 2}-b\left[\alpha+\left(w^{*} / c^{*}\right) \sqrt{\alpha^{2}+k^{2}\left(c^{* 2}-w^{* 2}\right)}\right]}{(\alpha-b)^{2}-k^{2} w^{* 2}-\left(w^{* 2} / c^{* 2}\right) b^{2}} v^{\prime}+i \frac{\alpha}{w^{*}} w^{\prime}+k u_{2}^{\prime} \\
-i \frac{v^{*}}{w^{*} c^{*}}\left[\frac{\left[(\alpha-b)^{2}-k^{2} w^{* 2}-\left(w^{*} / c^{*}\right)^{2} b r_{z}^{*}\right] \sqrt{\alpha^{2}+k^{2}\left(c^{* 2}-w^{* 2}\right)}}{(\alpha-b)^{2}-k^{2} w^{* 2}-\left(w^{* 2} / c^{* 2}\right) b^{2}}\right. \\
\left.-\frac{\left(w^{*} / c^{*}\right)\left(b-r_{z}^{*}\right)\left[\alpha(\alpha-b)-k^{2} w^{* 2}\right]}{(\alpha-b)^{2}-k^{2} w^{* 2}-\left(w^{* 2} / c^{* 2}\right) b^{2}}\right] p^{\prime} \\
+i \frac{r_{z}^{*}}{c^{* 2}}(\gamma-1) \Delta \epsilon \frac{\alpha(\alpha-b)-k^{2} w^{* 2}-b\left(w^{*} / c^{*}\right) \sqrt{\alpha^{2}+k^{2}\left(c^{* 2}-w^{* 2}\right)}}{(\alpha-b)^{2}-k^{2} w^{* 2}-\left(w^{* 2} / c^{* 2}\right) b^{2}} z^{\prime}=0
\end{gathered}
$$

Condition (100) represents the dispersion relation of the normal modes (70).

It is important to underline that the above dispersion relation shows a relevant influence of the chemical kinetics, due to the detailed form of the chemical reaction rate built in the hydrodynamic limit of the considered kinetic model. On the other hand, condition (100) recovers, as a particular case, the radiation condition used in several well known papers in the classical detonation literature [28, 29, 31, 33, 34], where a first-order reaction rate of Arrhenius form is adopted. More in detail, in this case, the partial derivatives $r_{v}^{*}$ and $r_{p}^{*}$ vanish at the end of the reaction zone so that from (94) it results $b=r_{z}^{*}$ and the above radiation condition simplifies to

$$
\begin{aligned}
& i \frac{\alpha}{w^{*}} w^{\prime}+k u_{2}^{\prime}-i \frac{v^{*}}{w^{*} c^{*}} \sqrt{\alpha^{2}+k^{2}\left(c^{* 2}-w^{* 2}\right)} p^{\prime} \\
& +i \frac{r_{z}^{*}}{c^{* 2}}(\gamma-1) \Delta \epsilon \frac{\alpha\left(\alpha-r_{z}^{*}\right)-k^{2} w^{* 2}-r_{z}^{*}\left(w^{*} / c^{*}\right) \sqrt{\alpha^{2}+k^{2}\left(c^{* 2}-w^{* 2}\right)}}{\left(\alpha-r_{z}^{*}\right)^{2}-k^{2} w^{* 2}-\left(w^{* 2} / c^{* 2}\right) r_{z}^{* 2}} z^{\prime}=0
\end{aligned}
$$

which is identical to the radiation condition derived in paper [31].

\section{Conclusions}

The interest of this paper is mainly oriented to investigate the equilibrium and stability properties of a detonation wave with reversible reaction, on the basis of a second order reaction rate deduced from the kinetic theory.

For the first time, the equation of the equilibrium Hugoniot curve is explicitly derived and then used to analytically define the equilibrium CJ-state and the corresponding CJ-velocity. As a result, the Hugoniot diagram of Fig. 1 for the study cases considered in Section 5 shows 
the correct location of points S and CJ, both belonging to the equilibrium Hugoniot curve. The wave structure problem is solved in two study cases proposed in Section 5. The numerical results appear to be consistent with the predictions advanced from the numerical studies and experimental works known in literature. Besides the already quoted papers, see for example Refs. [4, 35, 36] and Refs. [37, 38] for what concerns numerical studies and experimental results, respectively. Moreover, the response of the steady solution to the simulations widely reflects the resources incorporated in the mathematical model, especially those resources defining the chemical kinetics framework.

A second objective is addressed to the linear stability problem in presence of bidimensional perturbations, on the basis of the underlying steady solution. Adopting a normal mode approach, the mathematical analysis of Section 6 embraces the construction of the stability equations with their boundary conditions at the post-shock state, as well as the radiation condition at the end of the reaction zone. In particular, using an acoustic perturbation analysis, the radiation condition has been constructed in detail, and its final form shows that the chemical reaction gives several contributions to such closure condition, due to the kinetic description of the reaction mechanism.

In addition, an interesting aspect of such accurate picture is that the perturbation modes and the radiation condition in the case of a first-order reaction rate of Arrhenius type can be recovered as a particular case of the analysis developed here for a second-order reaction rate.

The stability analysis presented in this paper supplies the apparatus necessary to develop a computational treatment of the detonation stability. This will be the content of a forthcoming work.

\section{Acknowledgements}

The paper is partially supported by Brazilian Research Council (CNPq), by Italian Research Council GNFM-INdAM, and by the Research Centre of Mathematics of the University of Minho with the Portuguese Funds of FCT, Project PEstOE/MAT/UI0013/2014.

\section{References}

[1] J. H. S. Lee, The Detonation Phenomenon, Cambridge: Cambridge University Press, 2008.

[2] Cael G., Dick Ng H., Bates K. R., Nikiforakis N. and Short M., "Numerical simulation of detonation structures using a thermodynamically consistent and fully conservative reactive flow model for multi-component computations", Proc. R. Soc. A, 465, 2135$2153,2009$.

[3] Short M., "Theory and modeling of detonation wave stability: a brief look at the past and toward the future", 20th Int. Colloq. Dyn. Expl. React. Syst., 1-19, 2005.

[4] Faria L. M., Kasimov A. R. and Rosales R. R., "Study of a model equation in detonation theory", SIAM J. Appl. Math., 74, 547-570, 2014. 
[5] Conforto F., Groppi M., Monaco R., Spiga G., "Steady detonation waves for gases undergoing dissociation/recombination and bimolecular reactions", Continuum Mech. Thermodyn, 16, 149-161, 2004.

[6] Bisi M., Groppi M and Spiga G., "Flame structure from a kinetic model for chemical reactions", KRM, 3, 17-34, 2010.

[7] Conforto F., Monaco R. and Ricciardello A., "Analysis of steady combustion processes in a recombination reaction", Cont. Mech. Thermodyn., DOI 10.1007/s00161-013-0318-5, $1-17,2013$.

[8] Conforto F., Monaco R., Schürrer F. and Ziegler I., "Steady detonation waves via the Boltzmann equation for a reacting mixture", J. Phys. A: Math. Gen., 36, 5381-5398, 2003.

[9] Monaco R., Pandolfi Bianchi M. and Soares A. J., "A Kinetic Approach to Propagation and Stability of Detonation Waves", 26th International Symposium on Rarefied Gas Dynamics, AIP Conf. Proc., 1084, 45-50, 2009.

[10] Pandolfi Bianchi M. and Soares A. J., "Kinetic modelling and solutions to the linear stability of a detonation wave", J. Difference Eqs. Applic., 17, 1169-1184, 2011.

[11] Carvalho F. and Soares A. J., "On the dynamics and linear stability of one-dimensional steady detonation waves", J. Phys. A: Math. Theor., 45, 255501, 1-23, 2012.

[12] Lemarchand A., Nowakowski B., Dumazer G. and Antoine C., "Microscopic simulations of supersonic and subsonic exothermic chemical wave fronts and transition to detonation", J. Chem. Phys., 134, 034121, 1-11, 2011.

[13] Dumazer G., Leda M., Nowakowski B., and Lemarchand A., "Transition between an exothermic chemical wave front and a generic flame", Phys. Rev. E, 78, 016309, 1-6, 2008 .

[14] Kustova E. V. and Chikhaoui A., "Kinetic modelling of radiative reacting gas flow under strong nonequilibrium conditions", Chem. Phys., 255, 59-71, 2000.

[15] Aliat A., Chikhaoui A. and Kustova E. V., "Nonequilibrium kinetics of a radiative CO flow behind a shock wave", Phys. Rev. E, 68, 056306, 1-11, 2003.

[16] Marques Jr. W., Kremer G. M., and Soares A. J., "Influence of reaction heat on time dependent processes in a chemically reacting binary mixture", 28th International Symposium on Rarefied Gas Dynamics, AIP Conf. Proc., 1501, 123-144, 2012.

[17] Kremer G. M., An introduction to the Boltzmann equation and transport processes in gases Berlin: Springer, 2010.

[18] Fickett W. and Davis W. C., Detonation, Theory and Experiment, Berkeley: University of California, 1979.

[19] Fickett W., Introduction to Detonation Theory, Berkeley: University of California, 1986. 
[20] Wood, W. W. and Salsburg, Z. W., "Analysis of steady-state supported one dimensional detonations and shocks", Phys. Fluids, 3, 549-566, 1960.

[21] Muller I. and Ruggeri T., Rational Extended Thermodynamics Berlin: Springer, 1998.

[22] Ruggeri T. and Simić S., "Nonlinear wave propagation in binary mixtures of Euler fluids", Cont. Mech. Thermodyn., 16, 125-148, 2004.

[23] Simić S. and Ruggeri T., "Shock structure in a hyperbolic model of binary mixture of non-reacting gases", 1st Int. Congress of Serbian Society of Mechanics, 237-246, 2007.

[24] Ruggeri T. and Simić S., "On the hyperbolic system of a mixture of Eulerian fluids: a comparison between single- and multi-temperature models", Math. Meth. Appl. Sci., 30. 827-849, 2007.

[25] Sharpe G. J., "The structure of planar and curved detonation waves with reversible reactions", Phys. Fluids, 12, 3007-3020, 2000.

[26] Lax P. D., "Hyperbolic systems of conservation laws", Comm. Pure Appl. Math., 10, 537-566, 1957.

[27] Short M., "An asymptotic derivation of the linear stability of the square-wave detonation using the Newtonian limit", Proc. R. Soc. A, 452, 2203-2224, 1996.

[28] Lee H. I. and Stewart D. S., "Calculation of linear detonation stability: one dimensional instability of plane detonation", J. Fluid Mech. 216, 103-132, 1990.

[29] Short M. and Dold J. W., "Linear stability of a detonation wave with a model three-step chain-branching reaction", Math. Comput. Modelling, 24, 115-123, 1996.

[30] Sharpe G. J., "Linear stability of idealized detonations", Proc. R. Soc. A, 453, 26032625, 1997.

[31] Stewart D. S. and Kasimov A. R., "State of detonation stability theory and its application to propulsion", J. Propulsion and Power, 22, 1230-1244, 2006.

[32] Gorchkov V., Kiyanda C.B., Short M. and Quirk J. J., "A detonation stability formulation for arbitrary equations of state and multi-step reaction mechanisms", Proc. Combust. Instit., 31, 2398-2405, 2007.

[33] Short M. and Stewart D. S., "Low-frequency two-dimensional linear instability of plane detonation", J. Fluid Mech., 340, 249-295, 1997.

[34] Buckmaster J. D. and Ludford G. S. S., "The effect of structure on the stability of detonations I. Role of the induction zone", Proc. XX Simp. Comb., 1669-16761986.

[35] Taki S. and Fujiwara T., "Numerical analysis of two-dimensional nonsteady detonations", AIAA Journal, 16, 73-77, 1978.

[36] Oran E. S., Weber Jr J. W., Stefaniw. E. I., Lefebvre M. H. and Anderson Jr. J. D., "A numerical study of a two-dimensional $\mathrm{H}_{2}-\mathrm{O}_{2}$ - $\mathrm{Ar}$ detonation using a detailed chemical reaction model", Combust. Flame, 16, 147-163, 1998. 
[37] Cooper M., Jackson S., Austin J., Wintenberger E. and Shepherd J. E., "Direct experimental impulse measurements for detonations and deflagrations", J. Propulsion Power, 18, 1033-1041, 2002.

[38] Pintgen F., Eckett C. A., Austin J. M. and Shepherd J. E., "Direct observations of reaction zone structure in propagating detonations", Combust. Flame, 133, 211-229, 2003. 